\title{
Coverage Optimization Scheme Based on Artificial Fish Swarm Algorithm for Wireless Sensor Networks in Complicated Environment
}

\author{
Lei HONG*, Rui ZHONG \\ College of Information Technology, Jingling Institute of Technology, Nanjing 211169, \\ China \\ *honglei@jit.edu.cn
}

\begin{abstract}
With regard to the subject of sensor network node optimization, a strategy for wireless sensor network coverage optimization based on improved fish swarm algorithm is proposed in this paper. The improved algorithm is targeted on network coverage, node utilization rate and energy consumption balance, which makes use of the ergodic property of chaotic motion to overcome the disadvantage that artificial fish swarm algorithm may easily lead to regional optimization. As for this, the global searching ability of algorithm is improved and the solving efficiency is optimized. Moreover, the algorithm is able to adapt to complicated environment. Shown by related simulation experiment, the improved fish swarm algorithm could effectively optimize sensor network node deployment and improve network coverage rate. Compared with basic artificial fish swarm algorithm, improved fish swarm algorithm increases network coverage rate by $8.9 \%$.
\end{abstract}

Keywords: Wireless sensor networks, coverage optimal, artificial fish swarm algorithm

\section{Introduction}

Optimization of wireless sensor network is designed to reduce the number of nodes, and to improve network coverage rate [1-4] Both reducing node amount and improving network coverage are NP (Non-deterministic Polynomial) problems, which are hard to be solved with traditional optimization methods. Artificial fish swarm algorithm [5] model is simple, with strong ability to avoid regional optimization and rapid convergence to global optimization. Based on artificial fish swarm algorithm, the paper puts forward a deployment optimization strategy fitting for complicated environment, with the objective to maximize network coverage.

In recent years, with the development of swarm intelligent optimization algorithm, swarm intelligent algorithm is proved with good self-organizing and self-adapting ability, as well as strong robustness and expandability. On this basis, researches on wireless sensor network deployment algorithm based on swarm intelligent optimization algorithm have achieved significant results [6-8]. As a new swarm intelligent optimization algorithm, Artificial Fish Swarm Optimization (AFSO) is easy to be implemented, leading to strong adaptive ability in space searching. Moreover, the algorithm is not sensitive to initial parameter, with strong robustness, better convergence, and is able to acquire global extreme value, avoiding regional extreme value [9]. When being introduced into wireless sensor network deployment algorithm, artificial fish swarm algorithm may leads to better cluster structure, which extends 
the service life and reduces energy consumption of network. However, basic artificial fish swarm algorithm still needs to be improved in computing speed and amount [10-12].

Literature [13] puts to use basic artificial fish swarm algorithm to solve the mathematical model of wireless sensor network, with the aim to figure out the optimal coverage set of nodes in network. Basic artificial fish swarm algorithm is able to improve network coverage rate. However, the algorithm may also easily lead to regional optimal value, so that global optimal value becomes in accessible, affecting the coverage performance. Literature [14] employs artificial fish swarm intelligent optimization algorithm to improve network coverage of fixed position via global optimal direction angle. Literature [15] proposes a particle swarm optimization strategy based on probabilistic sensing model, realizing network coverage control based on basic particle sward optimization algorithm, with detailed instruction on the influence of sensing radius on coverage performance. The algorithm has certain optimization effect on WSN network coverage control. However, its solving speed is rather slow. Literature [16] combines chaotic motion with particle sward algorithm, overcoming the disadvantage of low solving speed, and avoiding the defect of easily leading to regional extreme value. However, the algorithm is over complicated, leading to complex solving process. Literature [17] realizes optimization and solution with genetic algorithm coverage model on regional energy consumption balance. Based on high coverage rate, the algorithm guarantees balanced network energy consumption. However, in region partition process, it consumes additional node energy. Literature [18] puts forward a mixed and hybrid genetic algorithm, which is able to reach precise solution efficiently. However, it doesn't take into consideration the energy of network node, and the computation is relatively complicated, which is unfavorable to sensor nodes with limited energy.

In this paper, in accordance with related features of wireless sensor, a coverage optimization strategy based on improved artificial fish swarm algorithm is proposed, with the objective to enlarge the coverage rate, to extend the service life of network, to shorten the running period, and to better optimize sensor network. The rest part of this paper is organized as follows: in Section 2, we propose the optimization model for wireless sensor networks. In Section 3, we optimize the scheme mentioned in Section 2 by fish-swarm algorithm. In Section 4, we simulate the coverage optimization scheme based on artificial fish swarm algorithm by using computer software and evaluate its performance. Finally, in Section 5, we reach the main conclusions.

\section{Wireless Sensor Network Optimization Model}

\subsection{Sensing prototype}

The operating environment of WSNs has significant influence on sensing and communication of nodes. Especially, WSNs are often applied in severe environment, so that the sensing and communication range of nodes may not be a circle with fixed radius. Sensing and communication are directional. Moreover, with the distance increases, the accuracy and probability of monitoring would be reduced accordingly, as is shown in Figure 1(a). This is often known as "sensing prototype". 


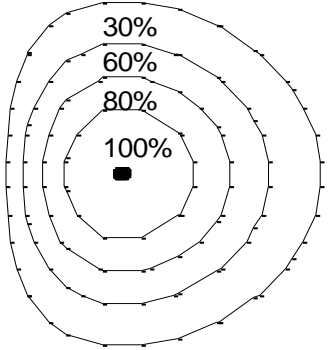

(a) Sensing prototype

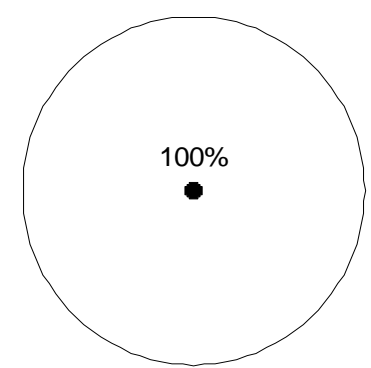

(b) Binary sensing model Figure 1. Sensing Model

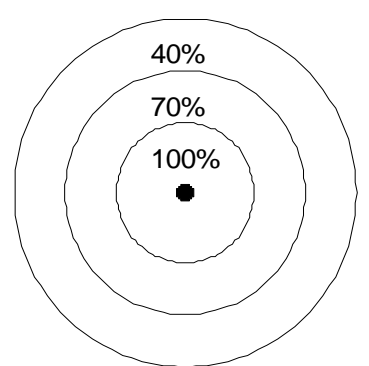

(c) Probability sensing model

\subsection{Node Sensing Model}

At present, there are mainly two kinds of node sensing models under researching in this field, i.e., Boolean Sensing Model (Fig. 1b) and Probabilistic Sensing Model (Figure 1c). The paper is aimed at node deployment in complicated environment, so that probability sensing model is applied, for it is more fitted for practical environment. Assuming that the target area $\mathrm{Z}$ to be monitored is a two-dimensional rectangle; wireless sensor network node set $\mathrm{S}=\left\{\mathrm{s}_{1}\right.$, $\left.\mathrm{s}_{2}, \ldots, \mathrm{s}_{\mathrm{n}}\right\}$, where, the coordinates of Node $\mathrm{s}_{\mathrm{i}}$ is $\left(\mathrm{x}_{\mathrm{i}}, \mathrm{y}_{\mathrm{i}}\right)$; As for this, the sensing probability of Node $s_{i}$ on a random point $\mathrm{p}$ in monitoring area $\mathrm{Z}$ shall be:

$$
C_{p}\left(\mathrm{~s}_{i}, \mathrm{p}\right)=\left\{\begin{array}{lc}
1, & d\left(\mathrm{~s}_{i}, \mathrm{p}\right) \leq R_{s}-R_{e} \\
e^{\left(\left(-\lambda_{1} \alpha_{1}{ }_{1}{ }_{1}\right) /\left(\alpha_{2} \beta_{2}+\lambda_{2}\right)\right)}, & R_{s}-R_{e}<d\left(\mathrm{~s}_{i}, \mathrm{p}\right)<R_{s}+R_{e} \\
0, & \text { otherwise }
\end{array}\right.
$$

In Equation (1), $\lambda_{1}, \lambda_{2}, \beta_{1}$, and $\beta_{2}$ are measuring parameters related sensors; $d\left(s_{i}, p\right)$ is the Euclidean distance between Node $s_{i}$ and a random point $p$ in the monitoring area; $R_{e}$ $\left(0<R_{e}<R_{s}\right)$ is measurement reliability parameter of sensor node; where, $\alpha_{1}$ and $\alpha_{2}$ satisfy: $\alpha_{1}=R_{e}-R_{s}+d\left(s_{i}, p\right), \alpha_{2}=R_{e}+R_{s}-d\left(s_{i}, p\right)$.

According to Equation (1), the joint monitoring probability of Node Set $\mathrm{S}$ on a random point $\mathrm{p}$ in Area $\mathrm{Z}$ shall be:

$$
C_{p}(\mathrm{~S}, \mathrm{p})=1-\prod_{i=1}^{n}\left(1-\mathrm{C}_{p}\left(\mathrm{~s}_{i}, \mathrm{p}\right)\right)
$$

In Area $\mathrm{Z}$, if the probability threshold of a random point's being effectively detected is denoted as $\mathrm{C}_{\mathrm{th}}$, then the below Equation (3) shall be true:

$$
\min _{x_{p}, y_{p}}\left\{C_{p}(\mathrm{~S}, \mathrm{p})\right\} \geq C_{t h}
$$

\subsection{Network Coverage}

Assuming that Monitoring Area $\mathrm{Z}$ may be digitally dispersed into $\mathrm{m} \times \mathrm{n}$ pixels, with the coordinates of pixel to be $(\mathrm{x}, \mathrm{y})$, while $\mathrm{N}$ sensor nodes with the same parameters are placed in the monitoring area; Assuming that the coordinates of each node are given, with the sensing radius to be $R_{s}$, and Communication Radius $R_{c}$ to be two times of Sensing Radius $R_{s}$, i.e., $R_{c}=2 R_{s}$. The ratio between coverage area of node set and total area of monitoring area is 
denoted as the regional coverage rate of node set, denoted as $F_{1}$. Hereby, the expression is described as (4).

$$
F_{1}=\frac{\sum_{x_{p}=1}^{m} \sum_{y_{p}=1}^{n} C_{p}(\mathrm{~S}, \mathrm{p})}{m \times n}
$$

Calculation steps of regional coverage rate $\mathrm{F}_{1}$ is shown below:

1) Based on Equation (1), the coverage rate of a pixel with regard to sensor node may be figured out;

2) Based on Equation (2), the joint coverage rate of a pixel with regard to sensor node set may be figured out;

3) Repeating (1) and (2) to work out the joint coverage rate of each pixel in monitoring area with regard to sensor node set;

4) Eventually, based on Equation (4), Regional Coverage Rate $F_{1}$ of sensor node set may be worked out.

Assuming $|\mathrm{N}|$ as the total amount of sensor nodes deployed in the network, and $\left|\mathrm{N}^{\prime}\right|$ as the number of operative sensor nodes so that Utilization Rate Function $\mathrm{F}_{2}$ of operative nodes in the network may be described as:

$$
F_{2}=\frac{|\mathrm{S}|}{\left|\mathrm{S}^{\prime}\right|}
$$

\subsection{Energy Balance}

Energy-saving is an important index to assess the performance of wireless sensor network. Energy-consumption balance of node is also an important index of energy-saving. Hereby, network energy balance function is given, shown as Equation (6).

$$
F_{3}=\frac{\operatorname{Max}\left(\mathrm{E}_{i}\right)-\mathrm{M} \operatorname{in}\left(\mathrm{E}_{i}\right)}{\frac{1}{n} \sum_{i=1}^{n} E_{i}}
$$

Where, $E_{i}$ refers to residual energy of node in the network; $F_{3}$ reflects the balancing degree of network energy consumption. Larger value indicates poorer energy consumption balance, i.e., the smaller, the better.

\subsection{Model Optimization}

On the basis of guaranteeing a certain coverage rate, we are to minimize operative node set as much as possible, so as to save energy, and to maintain balanced energy consumption. Weighted cooperation of Equation (4), (5) and (6) is taken as fitness functions for WSN coverage optimization in this paper, so that:

$$
F=w_{1} F_{1}+w_{2}\left(1-F_{2}\right)+w_{3}\left(1-F_{3}\right)
$$

Where, $\mathrm{w}_{1}, \mathrm{w}_{2}$ and $\mathrm{w}_{3}$ are weights, while $\mathrm{w}_{1}+\mathrm{w}_{2}+\mathrm{w}_{3}=1$. 


\section{Artificial Fish Swarm Algorithm Coverage Optimization}

\subsection{Classic artificial fish swarm algorithm}

Artificial fish swarm algorithm refers to an optimization method simulating fish behavior, which emerges in recent years. The algorithm imagines a swarm of "artificial fish", simulating natural fish's autonomous foraging behavior in water, so as to macroscopically show the advanced "artificial intelligence" behavior of fish swarm by individual's simple behavior and regional interaction. In the optimization process, individual's and swarm's regional optimization would tend to be closer to global optimization. Similar to particle swarm algorithm, ant colony algorithm and other relevant swarm algorithms, artificial fish swarm algorithm is also a random searching algorithm. As for this, there is no need for the algorithm to collect special information of problem. It only needs to compare the advantages and disadvantages according to target value, so that it is quite fitted for nonlinear function optimization. In addition, it has strong ability to avoid regional optimization, as well as rapid convergence.

Related definition of artificial fish: Decision-making Variable $\mathrm{X}=\left(\mathrm{x}_{1}, \mathrm{x}_{2}, \ldots, \mathrm{x}_{\mathrm{i}}\right)$ refers to present status of artificial fish; Target Function Value $\mathrm{Y}=\mathrm{f}(\mathrm{X})$ stands for food density of present position of artificial fish; $\mathrm{d}_{\mathrm{ij}}\left(\mathrm{X}_{\mathrm{i}}, \mathrm{X}_{\mathrm{j}}\right)$ represents the distance from Artificial Fish $\mathrm{X}_{\mathrm{i}}$ to Artificial Fish $\mathrm{X}_{\mathrm{j}}$; $\mathrm{S}$ is the motion step length of artificial fish; D refers to the factor of crowdedness; $\mathrm{R}$ is a random number from 0 to 1 . In addition, artificial fish is also configured with several optional behaviors: foraging behavior, rear-end behavior, swarm behavior and random behavior.

3.1.1. Foraging Behavior: Foraging behavior: simulating fish's random motion in water, seeking for food, and moving towards positions with high density of food. The present status of artificial fish is denoted as $X_{i}$. Then, a status $X_{j}$ is randomly selected within the sight range. If $Y_{j}>Y_{i}$, moving towards the direction for one step; or else, re-selecting Status $X_{j}$, and judging if it complies with the condition of moving forward. If the condition is still unsatisfied after several attempts, other behaviors will then be applied. The mathematical expression is described below:

$$
X_{i_{-} \text {next }}=X_{i}+R S \frac{X_{j}-X_{i}}{\left\|X_{j}-X_{i}\right\|}, \quad Y_{j}>Y_{i}
$$

3.1.2. Swarm Bhavior: Simulating fish's gathering behavior, i.e. moving towards the center of swarm; denoting the current status of artificial fish as $X_{\mathrm{i}}$, and exploring within the sight range the number of companions $n_{f}$ and the central position $X_{c}$; if $Y_{c}>Y_{i}$, and $Y_{c} / n_{f}>D Y_{i}$, there would be plenty of food at the center, and the center is not so crowded. As for this, moving towards the direction of $\mathrm{X}_{\mathrm{c}}$ for one step; otherwise, executing other behaviors. The mathematical expression is shown below:

$$
X_{i_{-} \text {next }}=X_{i}+R S \frac{X_{c}-X_{i}}{\left\|X_{c}-X_{i}\right\|}, \quad Y_{c}>Y_{i} \& Y_{c} / n_{f}>D Y_{i}
$$

3.1.3. Rear-end Bhavior: When there are other companions within the sight range, artificial fish may tend to be closer to companions with more food. Denoting the present status of artificial fish as $X_{i}$, the optimal companion within the searching sight range as $X_{\max }$, if $Y_{\max }>Y_{i}$, and the number of companions nf nearby $X_{\max }$ meets the condition of $Y_{\max } / n f>D Y_{i}$, the position of Companion $\mathrm{X}_{\max }$ hereby has more food, and is not so crowded. As for this, 
moving towards the direction of $\mathrm{X}_{\max }$ for one step; otherwise, executing other behaviors. The mathematical expression is shown below:

$$
X_{i_{-} \text {next }}=X_{i}+R S \frac{X_{\text {max }}-X_{i}}{\left\|X_{\text {max }}-X_{i}\right\|}, \quad Y_{\text {max }}>Y_{i} \& Y_{\max } / n_{f}>D Y_{i}
$$

3.1.4. Random Bhavior: Artificial fish randomly selects a behavior within its sight range, and then moves towards the direction. This is a default behavior in foraging behavior. In foraging behavior, when the number of repeats try_number is small, artificial fish would be provided with more change of random motion, which increases the diversification of swarm, so as to avoid regional extreme value.

3.1.5. Bulletin Bard: Bulletin board is a place recording the individual status of optimal artificial fish. After executing one behavior, each artificial fish would compare their present status with the status recorded on bulletin board. If their present status is superior to the status on bulletin board, they will replace the status on bulletin board with their present status. Otherwise, the status on bulletin board will not be changed. After iteration of the while algorithm is completed, value on bulletin board would be output as the optimal value.

3.1.6. Behavior Selection: In accordance with the nature of problem to be solved, each artificial fish is to assess the surrounding environment, so as to select a proper behavior for execution. For the problem of maximum value, the simplest way is to firstly simulating swarm and rear-end behavior, and then to assess the value of execution. The larger value would be chosen for execution. The default behavior is foraging behavior. Eventually, large volumes of artificial fish would gather at several regional extreme values, which is favorable for the acquisition of global extreme value field. Moreover, extreme value region with more optimal value often gathers plenty of artificial fish, which is helpful to the acquisition of global extreme value, so as to reach the goal of optimization.

\subsection{Improved AFSA}

Chaos is a special phenomenon in nonlinear dynamic system. Chaotic phenomenon has the below natures: 1) Randomness, i.e., the rule of chaotic phenomenon is greatly affected by initial value; 2) Ergodicity, i.e., it should be able to traverse all status without repetition, within a certain range, and according to its own rule. 3) Certainty, i.e. chaotic track is generated by ascertained iteration formula. As chaotic searching is easy to be executed, which may help to avoid regional extreme value, and is superior to random searching, it is quite advantageous in regional searching. Chaotic variable in the paper employs Tent projection [9, 10]:

$$
x_{i+1}= \begin{cases}2 x_{i} & x_{i} \in[0,0.5] \\ 2\left(1-x_{i}\right) & x_{i} \in(0.5,1]\end{cases}
$$

According to Tent projection, Artificial Fish i lead to chaotic point range in feasible region following the below steps:

1) Each dimensionality $X_{i k}(k=1 \ldots n)$ of artificial fish's status $X_{i}$ is projected into the interval $[0,1]$ according to Equation (12)

$$
c X_{i k}=\left(\mathrm{X}_{i k}-a_{k}\right) /\left(\mathrm{b}_{k}-a_{k}\right)
$$


Where, $a_{k}$ and $b_{k}$ separately represents the minimum value and maximum value of the $\mathrm{k}^{\text {th }}$ dimensionality variable $X_{\mathrm{ik}}$.

2) Iterating with Equation (11) for $M$ times to generate chaotic sequence $c X_{i k}^{1}, c X_{i k}^{2}, \ldots, c X_{i k}^{M}$;

3) Projecting the status value in chaotic sequence back to the original space according to Equation (13);

$$
X_{i k}^{s}=a_{k}+c X_{i k}^{s}\left(\mathrm{~b}_{k}-a_{k}\right)
$$

4) Based on these chaotic sequences, we will be able to figure out the chaotic point range of $X_{\mathrm{i}}$ upon Tent projection;

$$
X_{i}^{s}=\left(\mathrm{X}_{i 1}^{s}, \mathrm{X}_{i 2}^{s}, \ldots, \mathrm{X}_{i n}^{s}\right), \mathrm{s}=1, \ldots, \mathrm{M}
$$

5) Assessing the quality of the new status $x_{i}^{s}$ of artificial fish;

6) If the new status $X_{i}^{s}$ of artificial fish is superior to $X_{i}$, then, taking $X_{i}^{s}$ as the chaotic regional searching result; or else, denoting $s=s+1$, and returning to (2);

Chaotic searching is ergodic, so that it may be taken as an effective method to avoid regional optimization. Introducing the method into artificial fish swarm algorithm to realize global searching of artificial fish swarm algorithm; based on the result of artificial fish searching, chaotic regional searching is performed, so as to avoid that artificial fish disturbing around regional optimal value. As for this, the global convergence of artificial fish swarm algorithm is improved. Wireless sensor network coverage optimization algorithm based on chaotic searching is shown by Algorithm 1.

\section{Algorithm 1 Improved Artificial Fish Swarm Algorithm}

\footnotetext{
Algorithm: IAFSA

1) Initializing wireless sensor network, with each artificial fish representing one operative sensor node;

2) Initializing artificial fish parameter according to network scale and sensor node parameter, including position, maximum motion step length Step, visual range Visual, swarm scale n, crowdedness factor D, maximum iteration max_iterate and bulletin board.

3) Randomly generating $\mathrm{n}$ artificial fish in feasible region, and configuring initial iteration to be init_iterate $=0$;

4) Artificial fish is to calculate fitness function value according to Equation (7), and records status of all artificial fish on bulletin board when the calculated $\mathrm{f}$ value reaches the maximum.

5) Assessing the result when a certain artificial fish executes foraging behavior, rear-end behavior and swarm behavior; if after a certain behavior, the status of artificial fish is superior to present status, the artificial fish would hereby move towards the direction for one step, and then turning to 7);

6) All optimal artificial fish execute chaotic searching according to Equation (11), (12) and (13); The optimal artificial fish status within the solution range would consequently be figured out.

7) Updating the bulletin board, and recording the optimal artificial fish status in 7) on the bulletin board;

8) Judging algorithm ending condition: if reaching maximum iterations, ending the algorithm and outputting artificial fish status on bulletin board, i.e. the status of optimal wireless sensor network coverage; or else, init_iterate +1 , and turning to 5 ).
} 


\section{Simulation and Analysis}

\subsection{Simulation Environment and Parameter}

Assuming Monitoring Area $\mathrm{Z}$ of wireless sensor network is $50 \mathrm{~m} \times 50 \mathrm{~m}$, sensor node $\mathrm{N}=30$, sensing radius $R_{s}=50$, the improved fish swarm algorithm is employed to optimize wireless sensor network coverage rate in the monitoring area. The visual range of artificial fish is $10 \mathrm{~m}$, with step length to be 5m, try_number as 3, and crowdedness factor D as 0.6 . In order to test the effectiveness of the improved artificial fish swarm algorithm, wireless sensor network coverage optimized with basic artificial fish swarm algorithm is employed to be compared with the optimization algorithm proposed in this paper. Detailed simulation parameters are shown in Table 1:

\section{Table 1. Simulation Parameters}

\begin{tabular}{llll}
\hline Parameter Name & Parameter Value & Parameter Name & Parameter Value \\
\hline $\mathrm{Z}$ & $50 \mathrm{~m} \times 50 \mathrm{~m}$ & $\mathrm{~N}$ & 30 \\
$\mathrm{R}_{\mathrm{s}}$ & $5 \mathrm{~m}$ & $\lambda_{1}$ & 1.0 \\
$\mathrm{R}_{\mathrm{c}}$ & $10 \mathrm{~m}$ & $\lambda_{2}$ & 0 \\
$\mathrm{R}_{\mathrm{e}}$ & $2 \mathrm{~m}$ & $\beta_{1}$ & 1.0 \\
$\mathrm{E}_{\mathrm{i}}$ & $0.5 \mathrm{~J}$ & $\beta_{2}$ & 1.5 \\
$\mathrm{M}$ & 50 & $\mathrm{w}_{1}$ & 0.6 \\
$\mathrm{c}_{\mathrm{th}}$ & 1.0 & $\mathrm{w}_{2}$ & 0.3 \\
Step & $5 \mathrm{~m}$ & $\mathrm{w}_{3}$ & 0.1 \\
Visual & $10 \mathrm{~m}$ & max_iterate & 100 \\
try_number & 3 & $\mathrm{D}$ & 0.6 \\
\hline
\end{tabular}

\subsection{Simulation Results}

As is shown in Figure 1, circles refer to the sensing range of sensor nodes, while dots are position of sensor nodes.

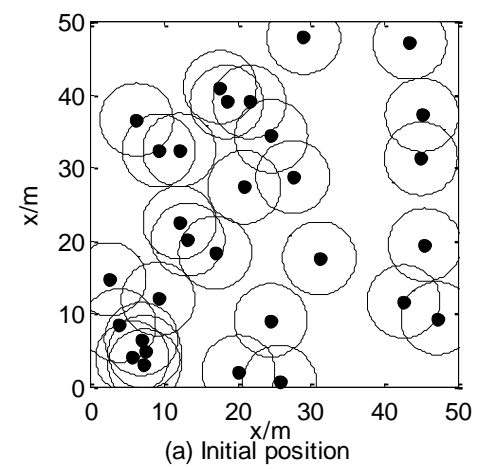

(a) Initial position

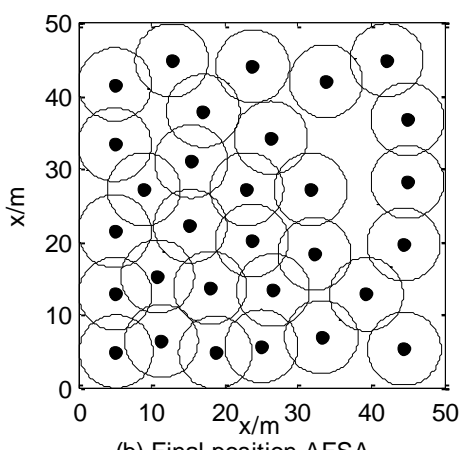

(b) Final position AFSA

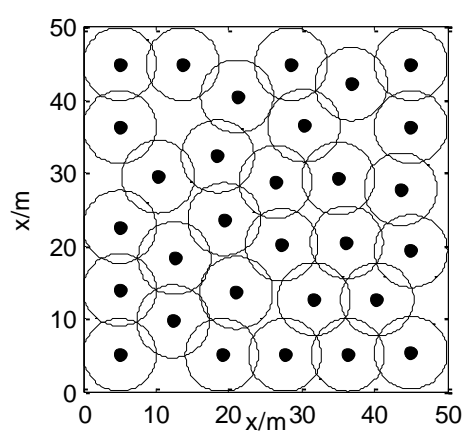

(c) Final position IAFSA

Figure 1. Comparison of Network Coverage between AFSA and IAFSA

The optimization result of basic artificial fish swarm algorithm is $81.3 \%$. By contrast, the improved artificial fish swarm algorithm may improve network coverage rate up to $90.2 \%$. It may as well be seen from Fig. 1 that, optimized by the latter algorithm, network nodes are evenly distributed in the whole target area.

In addition, in order to test the performance of IAFSA algorithm in complicated environment, Coverage [21] and Uniformity [22] are selected as the indices to evaluate its 
performance. Numerous simulations have been carried out in various environments, including deployment in area with obstructs, deployment in region of interest, as well as re-deployment upon node failure.

The red rectangle indicates the size of the region (area Z). Tiny black circles represent the positions of nodes; small (shaded) and large circles are used to indicate the sensing radius and the communication radius, respectively. Nodes can probe the environment and collect information in the sensing radius Rs. Similarly, it can be influenced by its adjacent nodes and repulsive force from the obstacles as well. The communication radius of the node $R_{c}$ is greater than the sensing radius $R_{s}$. Nodes are able to exchange information with other nodes within its communication radius.

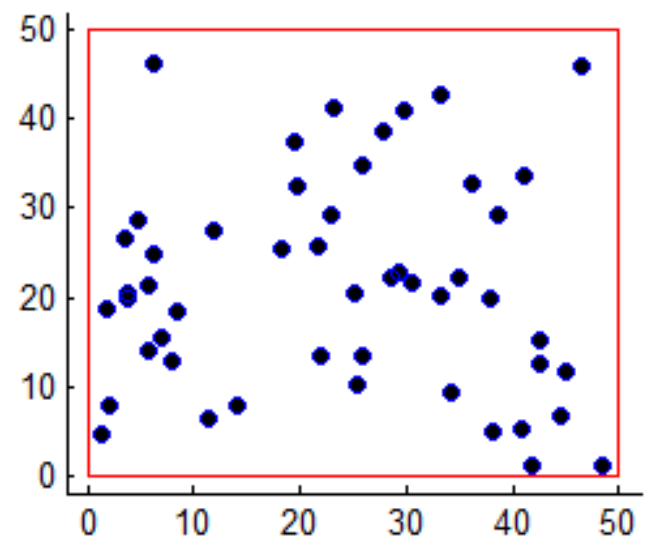

Figure 2. Initial Deployment

When starting deploying, all nodes move to fill the entire region, and eventually reach equilibrium, as in positions shown in Figure 3. The right plot shows the change in Coverage and Uniformity versus iteration during the deployment process described in the left plot. The $y$-coordinate on the left is the uniformity while the right represents the coverage. We can see from the right plot that, at the initial moment of deployment, the nodes can only cover a small part of the region because they are all initially positioned randomly. Therefore the coverage is practically low $(<30 \%)$. With time elapsed and all nodes are in motion, the coverage tend to grow with time, and reach equilibrium at a certain moment (about Iteration=60). This is when the coverage reaches maximum value and maintains at that until the simulation is over. The value of uniformity is initially around 0.7 . Then it starts to fall back quickly. The uniformity eventually tends to be around zero. Because uniformity is represented using standard deviations, so the smaller it is, the more uniform the network is. 

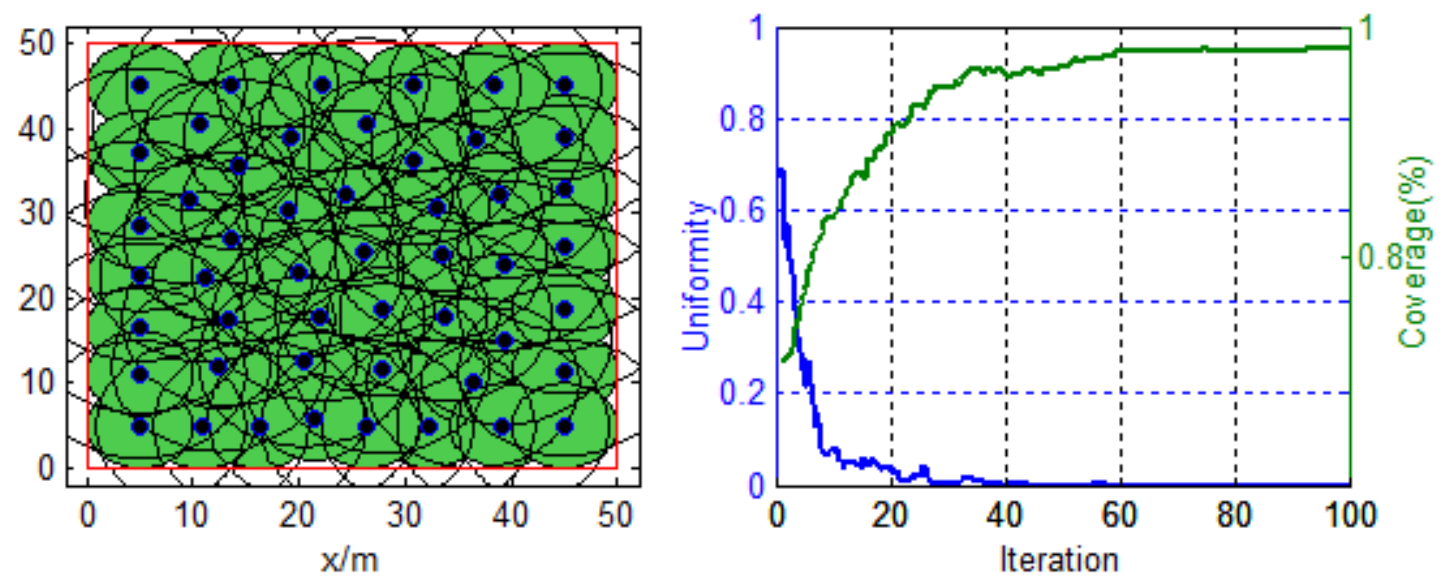

Figure 3. Normal Deployment

In the left plot in Figure 4, the red circle represents obstacles. There are 2 obstacles in the left plot. From the left plot, the nodes will be deployed at a distance with the obstacle to avoid them, due to the repulsive force from the obstacles. This is highly meaningful in practical environment. To keep the nodes away from hazard or unreachable region can minimize node damage, indicates the algorithm's adaptivity. The right plot shows the change in coverage and uniformity versus time during the deployment process. We can see that because of the obstacles, the region is not completely covered with nodes. The maximum of coverage is less than 1. In the meantime, compared with Figured 3, the value of uniformity is higher after nodes have reached equilibrium. That is due to the existence of the obstacles.
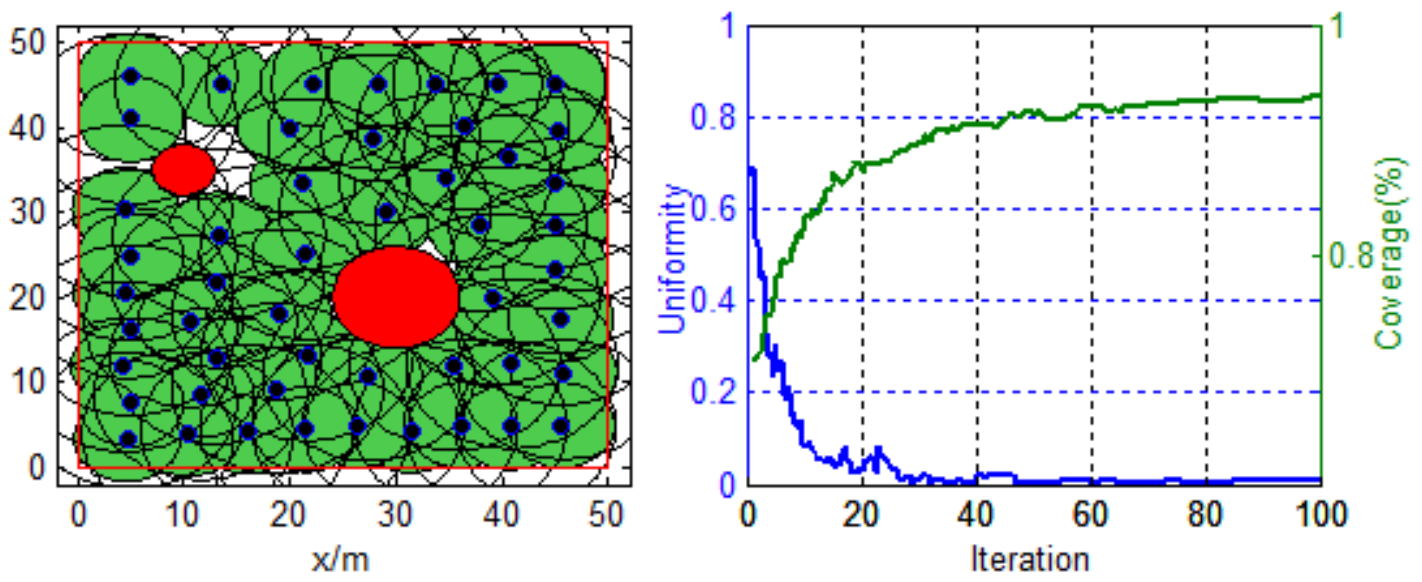

Figure 4. Deployment with Obstacles

In Figure 5, the red dots represent disabled nodes. The deployment process is consisted of two stages. In stage 1 , nodes are in deployed completely with regular circumstance (Iteration=100), reached equilibrium. In stage 2, some nodes are disabled due to environmental or other factors in simulation. There are 5 node failures shown in the figure. These node failures will break the equilibrium of the network. Nodes are being re-deployed to fill the "blanks" due to the failed nodes. Nodes are moving toward failed nodes to cover the "exposed" areas. After slight adjustments, nodes have reached equilibrium once again, as shown in the left plot. Based on the Coverage and Uniformity curve in the right plot, we can 
learn the process clearly. At Iteration $=100$ (where the arrow points), there's significant decrease in coverage rate. In the meantime, the index for Uniformity has risen slightly. These are all caused by node failures. After the re-deployment $(100<$ Iteration<200), nodes are in new equilibrium. Coverage is back to some value close to 1 . Yet the uniformity is higher than the previous value. These are caused by the decrease in uniformity due to node failures.
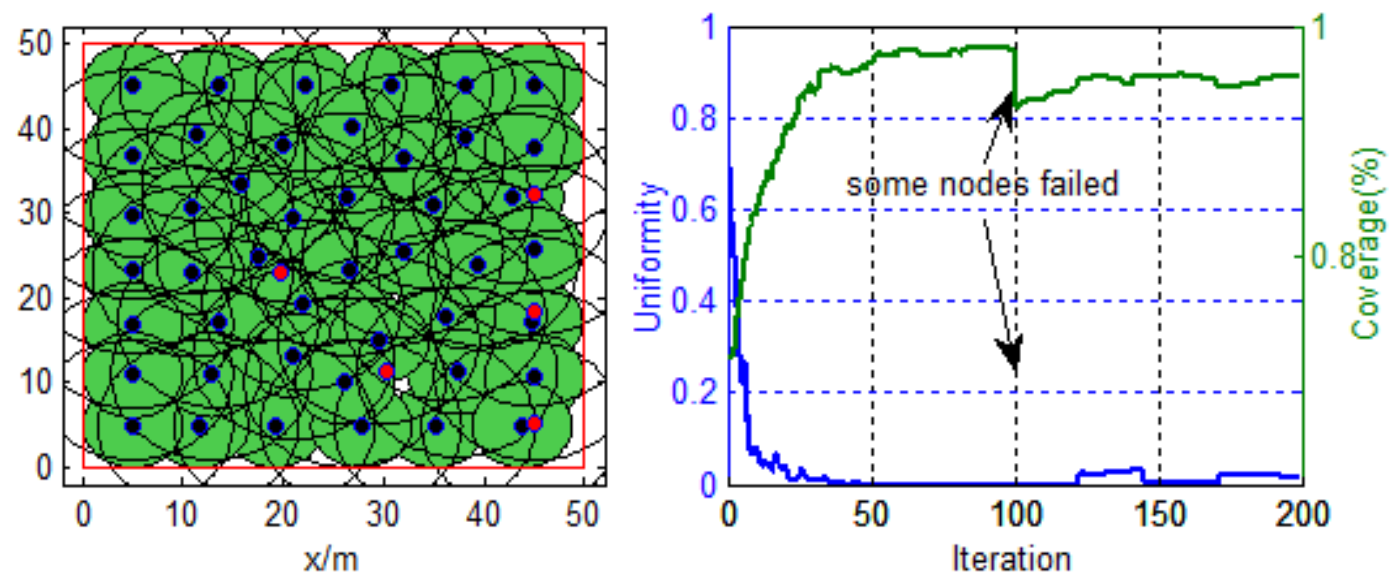

Figure 5. Re-deployment after Node Failures

In Figure 6, the blue region in the left plot represents region of interest (ROI). ROI is the region where nodes need to be deployed with emphasis, because these are usually the monitoring focus. There are two ROI's, judging from the figure. The one in lower left corner is relatively small, while the upper right one is larger. It can be seen that, after the deployment, there are more nodes gathered around or in ROI's, meaning ROI's have higher node densities, so that ROI's are better covered and served. The Coverage and Uniformity curve in the right plot indicates the change in Coverage and Uniformity. From the plot, after the equilibrium is reached, coverage is slightly less than that in normal deployment (shown in Figure 3). That is caused by the attraction of nodes by the ROI's. The value of Uniformity is slightly larger than that in Figure 3, which means that the uniformity when deploying with ROI's is slightly worse than that in normal deployment.
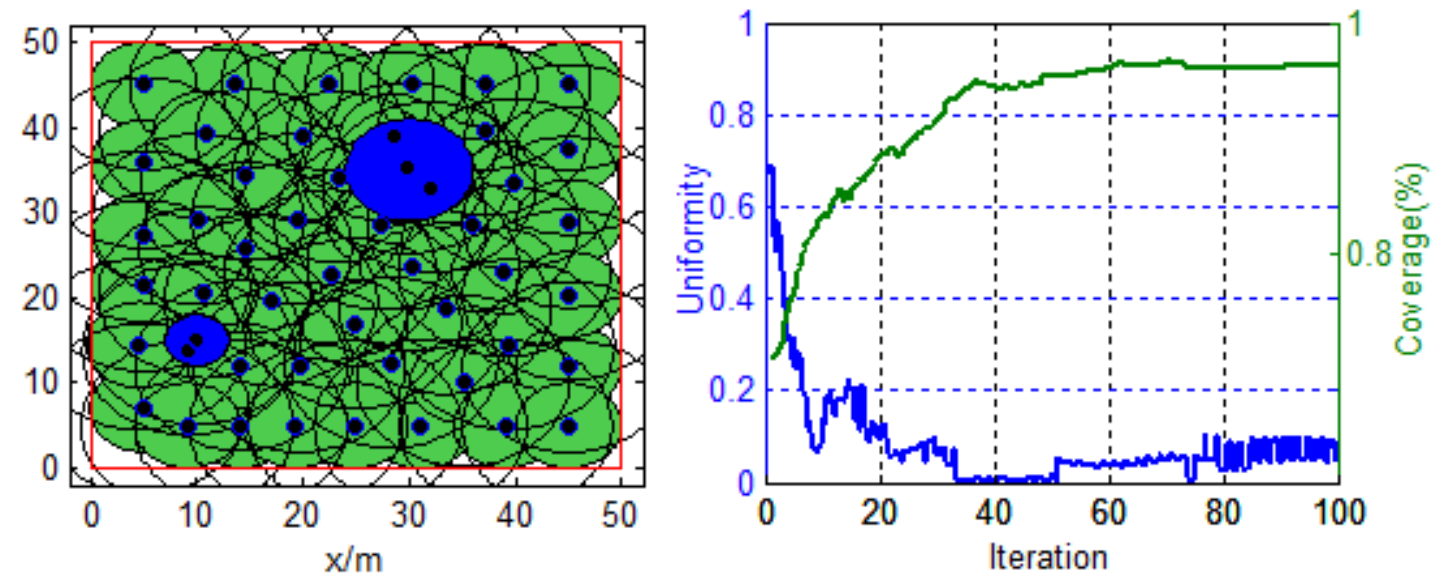

Figure 6. Deployment with Region of Interest (ROI) 
Based on the above simulation, the result shows that the improved artificial fish swarm algorithm is able to enhance the exploration ability and searching efficiency of fish swarm in space, improving the solving precision, and is helpful in figuring out approximate optimal coverage node set, with better sensor network optimization effect.

\section{Conclusion}

With regard to the subject of sensor network optimization, a coverage optimization strategy based on improved fish swarm algorithm is proposed in this paper. Network energy balance calculation, chaotic searching and artificial fish swarm algorithm are combined and applied in WSN coverage optimization problem, with improved fish swarm WSN coverage optimization algorithm proposed. The algorithm puts to use node probability sensing model more fitted for practical environment, taking network coverage rate, node utilization ratio and energy consumption balance as the optimization objective, so as to implement chaotic searching on nodes, preventing nodes from regional optimization, separating nodes from disturbing around global optimization in later stage, so as to ensure the precision and convergence of the algorithm. By comparing with basic artificial fish swarm algorithm, the algorithm proposed in this paper is proved with better coverage rate, evenness, precision and efficiency, leading to better WSN coverage optimization effect.

\section{References}

[1] C. D. Scott and R. E. Smalley, "Nanosci, Nanotechnol", (2003), pp. 3-75.

[2] C. Ozturk, D. Karaboga and B. Gorkemli, "Sensors", (2011), pp. 11- 6.

[3] C. Zhu, C. Zheng, L. Shu and G. Han, "Journal of Network and Computer Applications", (2012), pp. 2, 35 .

[4] G. Tuna, V. C. Gungor and K. Gulez, “Ad Hoc Networks”, (2014), pp. 13.

[5] X. Li, F. Lu, G. Tian and J. X. Qian, Journal of Shandong University, vol. 34, no. 5, (2004).

[6] Q. Bai, "Computer \& Information Science", vol. 3, no.1, (2010).

[7] K. E. Parsopoulos and M. N. Vrahatis, "Particle swarm optimization and intelligence: advances and applications Hershey: Information Science Reference", (2010).

[8] R. S. Parpinelli and H. S. Lopes, International Journal of Bio-Inspired Computation, vol. 3, no.1, (2011).

[9] W. Yiyue, L. Hongmei and H. Hengyang, "Wireless sensor network deployment using an optimized artificial fish swarm algorithm", Computer Science and Electronics Engineering (ICCSEE), (2012), March 23-25, Hangzhou, China.

[10] X. Min, S. Wei-Ren, J. Chang-Jiang and Z. Ying, AEU-International Journal of Electronics and Communications, vol. 64, no. 4, (2010).

[11] A. Chamam and S. A. Pierre, "Computers \& electrical engineering", vol. 36, no. 2, (2010).

[12] K. Akkaya, F. Senel and B. McLaughlan, Journal of Parallel and Distributed Computing, vol. 69, no. 6, (2009).

[13] H. Yuyue and L. Keqing, "Application Research of Computers”, vol. 30, no. 2, (2013).

[14] K. Zhang, W. Zhang, C. Dai and J. Z. Zeng, "Optoelectronics letters", (2010), pp. 6.

[15] L. Zhuliang and F. Yuanjing, "Computer Simulation”, vol. 4, (2009).

[16] L. Weiting and F. Zhouyuan, "Journal of Computer Applications", vol. 31, no. 2, (2011).

[17] W. Jianhua, S. Mingyue and F. Youbing, "Computer Simulation", vol. 29, no. 2, (2012)

[18] L. Meijin, S. Caihong and W. Fei, Computer Simulation, vol. 28, no. 3, (2011)

[19] J. Zhao, "Chaotic particle swarm optimization algorithm based on tent mapping for dynamic origindestination matrix estimation", Electric Information and Control Engineering (ICEICE), (2011) April 15-17, Wuhan, China.

[20] K. Zhu and M. Jiang, "An improved artificial fish swarm algorithm based on chaotic search and feedback strategy", Computational Intelligence and Software Engineering, (2009) December11-13, Wuhan, China.

[21] D. W. Gage, "Command control for many-robot systems", (1992).

[22] N. Heo and P. K. Varshney, "A distributed self spreading algorithm for mobile wireless sensor networks", WCNC, New Orleans, (2003) March 20-20, LA, USA. 


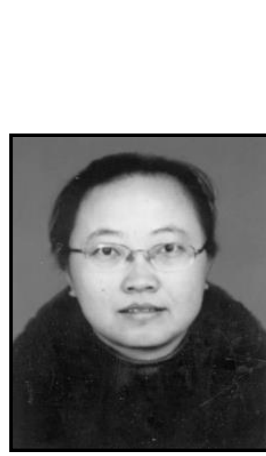

\section{Authors}

Lei Hong, she received the Bachelor Degree from Anhui Normal University in 1998, the Master. Degree from Hohai University in 2005. From 2004 to now, she is a lecturer at the School of Information Technology, Jinling Institute of Technology. Her current research interests include Software Engineering and Wireless Sensor Network.

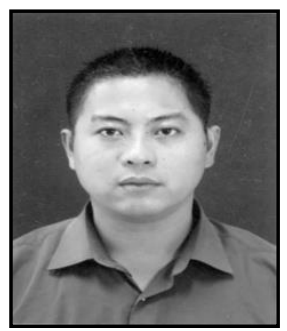

Rui Zhong, he received the Bachelor Degree from Changchun University of Science and Technology in 1997, the Master. Degree from East China Normal University in 2006. From 2003 to now, he is a lecturer at the School of Information Technology, Jinling Institute of Technology. His current research interests include Software Engineering. 
International Journal of Future Generation Communication and Networking Vol.7, No.5 (2014) 